\title{
USING ESSENTIAL OILS TO COMBAT THE THREAT OF MULTI-DRUG RESISTANT BACTERIA, PSEUDOMONAS AERUGINOSA
}

\author{
JENIES GRULLON ${ }^{1}$, JAMES P. MACK ${ }^{1}$, ALBERT ROJTMAN ${ }^{2}$ \\ ${ }^{1}$ Department of Biology, Monmouth University, West Long Branch, NJ, ${ }^{2}$ Department of Pathology, Meridian Health, Jersey Shore University \\ Medical Center, Neptune, NJ \\ Email: mack@monmouth.edu
}

Received: 16 Aug 2016 Revised and Accepted: 05 Oct 2016

\begin{abstract}
Objective: The development of antibiotics was a revolutionary scientific discovery and medical advancement that greatly extended the life expectancy of mankind. Through less than $100 \mathrm{y}$ of using antibiotics to treat infectious bacteria, some of these highly adaptive organisms have developed resistance to the drugs. The healthcare field is greatly concerned with the threat of many common infections that have been considered treatable for decades, regaining its ability to be severely fatal; thus, making alternative treatments currently in high demand. This study concentrated on investigating an alternative treatment for a specific gram-negative bacterium, Pseudomonas aeruginosa ( $P$. aeruginosa), a resistance-gaining bacteria that commonly infects hospitalized patients with weakened immune systems and/or open wounds.
\end{abstract}

Methods: Prior to the age of modern medicine, human beings relied on nature for medicinal treatments. In our research, we focused on determining the in vitro efficacy of using the essential oils, cassia and cinnamon bark, their major component, cinnamaldehyde, as well as the major component of manuka honey, methylglyoxal, as an alternative treatment against $P$. aeruginosa We tested cassia, cinnamon bark, cinnamaldehyde, and methylglyoxal using the Kirby-Bauer disk diffusion method; the diameter of the zone of inhibition for each treated bacterial sample was measured and compared with the standard antibiotic treatments, tobramycin, and amikacin.

Results: This study showed that the selected essential oils, cinnamaldehyde, and methylglyoxal were as effective or better in inhibiting the growth of $P$. aeruginosa compared to the standard aminoglycoside antibiotics.

Conclusion: The tested essential oils, cinnamaldehyde, and methylglyoxal may be useful as an alternative treatment for infections caused by $P$. aeruginosa and may also provide communities where antibiotics are not readily available, a cost-effective way to treat this infectious disease.

Keywords: Essential oils, Multi-drug resistant, Pseudomonas aeruginosa, Cinnamon, Cassia, Methylglyoxal, Antibiotic resistance.

(C) 2016 The Authors. Published by Innovare Academic Sciences Pvt Ltd. This is an open access article under the CC BY license (http://creativecommons.org/licenses/by/4. 0/) DOI: http://dx.doi.org/10.22159/ijpps.2016v8i12.13564

\section{INTRODUCTION}

The history of using natural plant products in medicinal practices dates back to 4,500 B. C. in Ancient Egyptian civilizations. It is then that medicinal oils extracted from a variety of plants were first recorded as the treatment for various human maladies $[1,2]$. Today, antibiotics, synthetically produced or naturally occurring chemicals, are amongst the most commonly prescribed drugs in the world, effective in treating pathogenic bacterial infections. Not very long ago, in the early $20^{\text {th }}$ century, pneumonia, tuberculosis, diarrhea, and diphtheria were among the leading causes of death [4]. Due to the development of antibiotics mortality rates by these diseases and other bacterial infectious diseases have been significantly reduced while dramatically increasing human life expectancy. For that reason, scientists and medical professionals alike, consider antibiotics as one of the most revolutionary developments in human history [2, 5].

Unfortunately, the success of this class of drugs has been accompanied by the rapid growth of antibiotic-resistant bacterial strains. These strains have emerged due to the widespread use, overuse and misuse of antibiotics along with the opportunistic nature of these pathogenic organisms [5]. Over-prescription, incorrect dosage, lack of patient compliance as well as agricultural and other consumer industry applications of this class of drugs have all contributed to the increased threat of antibiotic resistance [3]. Various biochemical and physiological mechanisms allow these small organisms to develop and retain resistance [5].

Antibiotic resistance is a complex problem with the power to have catastrophic consequences on our very way of life. It is a significant global healthcare threat that has the potential to return us to the pre-antibiotic era where mortality due to infectious diseases was much greater $[3,5,6]$. The United States government has acknowledged antibiotic resistance as a global crisis and has set out initiatives to work nationally and internationally to prevent and control death due to this resistance [7]. The emergence of newer multi-drug resistant bacterial strains has continued its relentless growth [5] and research to find suitable solutions continue. Today, antibiotics continue to serve as the primary treatment for infectious diseases but plants used centuries ago for their antibacterial properties may serve as our salvation and return to the medical field to combat multi-drug resistance [8].

In this, in vitro study, an alternative method of treating infections caused by the multi-drug resistant bacterium, P. aeruginosa, was explored. P. aeruginosa is gram-negative, aerobic, bacillus bacterium that has over time developed resistance to multiple antibiotics. The bacterium is found widely in the environment but has become a health problem in hospital settings [9]. P. aeruginosa most commonly causes pneumonia, infections of the bloodstream, urinary tract infections, swimmers ear infections, as well as surgical site and burn site infections [3]. P. aeruginosa commonly infects those patients with weakened immune system, and if left untreated can lead to severe illness and even death. 51,000 healthcare-associated drug-resistant P. aeruginosa infections result in roughly 400 deaths per year [3].

$P$. aeruginosa infections are most effectively treated with aminoglycosides including amikacin, gentamicin,-streptomycin, tobramycin and neomycin which interfere with the 30 subunit during protein synthesis in the bacterium. However, P. aeruginosa has shown an increase in resistance to these once most effective antibiotics $[1,6$, $8,10]$. Physicians are running out of antibiotics to treat these serious multi-drug resistant bacterial infections, particularly those caused by gram-negative bacteria like $P$. aeruginosa $[3,8]$. Gram-negative bacteria have an outer membrane, making the inhibition of its growth significantly more challenging [3]. The outer membrane contains 
various proteins as well as lipopolysaccharides (LPS). The LPS, which is composed of a lipid, a core polysaccharide, and a highly variable 0 antigen, forms an extra barrier in gram-negative bacteria to make them more resistant to growth inhibitors [1]. Of the most threatening gram-negative pathogens, $P$. aeruginosa ranks second, with a $58 \%$ mortality rate [10].

The developments of new strategically designed and effective antibiotics have been limited. Even if developed, such new drugs may only serve to temporarily manage the treatment or control of multi-drug resistance bacterial infections [3] because the ability of bacteria to develop resistance to the traditional antibiotics will persist. Therefore, the demand for developing and proving alternative antimicrobial therapies is high [12,13]. Examples of alternative treatments that have continuously demonstrated their efficacy and thus earning respect in the medical field are essential oils [14] and honey [12]. Essential oils are contained within granular cells of a variety of plant's components including seeds, bark, roots, leaves, flowers, wood, balsam and resin; these parts are all responsible for the particular smell and flavor associated with each plant [14]. Essential oils are extracted from aromatic medicinal plants using conventional techniques such as distillation [2]. The composition of essential oils was determined to be complex mixtures of chemicals including various alcohols, aldehydes, terpenes, ethers, ketones, phenols and oxides [2]. Honey is commonly used in wound dressing because of its reputation for having antibacterial properties [11]. The two antimicrobial components identified as being responsible for the effectiveness of honey are hydrogen peroxide and phytochemical components $[7,12]$.

Our research studied the in vitro antibacterial effects of the essential oils cassia and cinnamon bark (their primary component is cinnamaldehyde) and the major component of manuka honey (Leptospermum scoparium), methylglyoxal [14] on an ATCC strain of $P$. aeruginosa. These compounds were tested as emollients using lanolin and jojoba oil as carriers. Methylglyoxal and cinnamaldehyde both contain functional group aldehyde, which is known to be responsible for the antibacterial properties of some essential oils. The antimicrobial activity of essential oils and their components is likely due to their ability to inhibit or interact with multiple targets of the cell, including their membranes and cytoplasmic structures [2]. Their hydrophobic properties facilitate their penetration into cell membranes causing the alteration to the structure of membranes as well as leakage of cell contents and sometimes completely change the morphology of the organism's cells $[1,2]$.

\section{MATERIALS AND METHODS}

\section{The drug-resistant bacterial sample}

The bacterial sample used for this research was $P$. aeruginosa (Schroeter) Migula (ATCC ${ }^{\circledR} 27853^{\text {TM }}$ ).

\section{Supply of standard antibiotics}

The standard antibiotics implemented in this study were obtained from BD (Becton, Dickinson, and Company) NJ, USA and are listed below:

\section{- Amikacin Susceptibility Test Discs $30 \mu \mathrm{g}-\mathrm{BD}$ BBL ${ }^{\mathrm{TM}}$}

- Tobramycin Susceptibility Test Discs $5 \mu \mathrm{g}-\mathrm{BD}_{\mathrm{BBL}}^{\mathrm{TM}}$

\section{Essential oils and components}

Obtained from dōTERRA ${ }^{\circledR}$ Essential Oils of West Pleasant Grove, Utah were the following essential oils: cassia and cinnamon. According to dōTERRA®Essential Oils obtains all of their essential oils were obtained via the steam distillation method. The chemical components trans-Cinnamaldehyde, (99\%) and methylglyoxal (40\% in $\mathrm{H}_{2} \mathrm{O}$ ) were both obtained from Sigma-Aldrich ${ }^{\circledR}$ Missouri, USA.

\section{The emollients}

The carrier oils used in this study were Jojoba Oil from SigmaAldrich ${ }^{\circledR}$ Missouri, USA and Liquid Lanolin from Now ${ }^{\circledR}$ Solutions, Bloomingdale, IL, USA.

\section{Preparation of medium}

The medium used to provide a stable and supportive environment for the $P$. aeruginosa during the susceptibility tests, as well as promote its growth during this study was BD Difco ${ }^{\text {TMMueller Hinton }}$ II Agar. The agar was prepared using dehydrated powder following the manufacturer's instructions. Subsequently, the formed agar was properly autoclaved and poured into sterile Petri dishes to solidify.

\section{Use of the kirby-bauer disk diffusion method}

Following the isolated culturing of $P$. aeruginosa samples, a disk diffusion method was used to measure the zones of inhibitions caused by the compounds applied. The cultures of $P$. aeruginosa were grown to match a $1 \times 10^{8}$ Colony Forming Units per milliliter $(\mathrm{ml})$ in $0.5 \mathrm{McF}$ arland standard test tube with tryptic soy broth.

The $P$. aeruginosa cultures were then streaked onto the solidified Mueller Hinton II agar plates for optimum growth. The antibacterial activity of the specific five (5) $\mu \mathrm{l}$ combinations of methylglyoxal, cinnamaldehyde, essential oils, and emollients were applied directly to sterile blank paper discs $(6 \mathrm{~mm}) \mathrm{BD}$ Difco $^{\mathrm{TM}}$ using Positive Displacement Pipettes from Ranin ${ }^{\mathrm{TM}}$ Instruments, San Diego, CA. These saturated discs were then applied to the middle of the streaked Petri dishes. Similarly, the standard antibiotic amikacin and tobramycin susceptibility test discs were positioned onto the center of streaked Petri dishes. After incubating at $37{ }^{\circ} \mathrm{C}$ for approximately $24 \mathrm{~h}$, the diameter of the zones of inhibition for each triplicate set was measured, averaged, and compared with that of the standard antibiotics used in a clinical setting [15].

\section{RESULTS AND DISCUSSION}

Cassia and cinnamon essential oils, their major component cinnamaldehyde, as well as methylglyoxal, derived from manuka honey, were all tested for their ability to inhibit the growth of multidrug resistant $P$. aeruginosa in this study. Realistically, because essential oils and their components cause skin irritation when applied directly to the skin, diluting these compounds is necessary for their dermal application. In this in vitro study emollients were prepared by diluting the essential oils and chemicals in lanolin and jojoba oils as carriers. In order to ensure that the carrier oils themselves were not having an antimicrobial effect on the $P$. aeruginosa, they were tested at $100 \%$ concentration first. Results from these experiments showed that lanolin and jojoba oils had no antimicrobial properties inhibiting the growth of $P$. aeruginosa (table 1). These results ensured that the zones of inhibition observed in the tests using dilutions with carrier oils could solely be attributed to the properties of essential oils or ability of chemical components to act as an antimicrobial agent.

Table 1: Mean diameter of zone of inhibition of $100 \%$ carrier oils: lanolin and jojoba oil

\begin{tabular}{ll}
\hline Carrier oil & Mean diameter of zone of inhibition (mm) \\
\hline Lanolin & 0 \\
Jojoba oil & 0 \\
\hline
\end{tabular}

The zones of inhibition of dilutions at various concentrations were tested to determine the emollient's minimum inhibitory concentration (MIC). At the MIC, the emollient(s) tested demonstrated results that were similar or greater in its effectiveness as the standard antibiotics measured. Before being able to determine the MIC for the emollients, the zone of inhibition of the standards (antibiotics, tobramycin, and amikacin) were found (table 2).

Table 2: Mean diameter of zone of inhibition

\begin{tabular}{ll}
\hline Antibiotic & Mean diameter of zone of inhibition (mm) \\
\hline Amikacin & 21 \\
Tobramycin & 23 \\
\hline
\end{tabular}


The compounds were diluted at $25 \%, 50 \%$, and $80 \%$ concentrations; results demonstrated the MIC to be $80 \%$ (table 3 and fig. 1). Essential oils are known to work less efficiently on gram-negative bacteria due to the hydrophilic outer membrane [3] which may explain the high observed MIC compared to experiments with grampositive organisms. The experiment was performed in triplicates under aseptic conditions; measurement of zones of inhibitions of each set was averaged to yield a mean diameters zone of inhibition.

Table 3: Mean diameter of zone of inhibition of cassia, cinnamon, methylglyoxal, and cinnamaldehyde at various dilutions in lanolin and jojoba oil

\begin{tabular}{lll}
\hline Essential oil (concentration & Carrier oil & Mean diameter of zone of inhibition (mm) \\
\hline Cassia $(80 \%)$ & Lanolin & 25 \\
& Jojoba oil & 26.5 \\
Cassia $(50 \%)$ & Lanolin & 16 \\
& Jojoba oil & 21 \\
Cassia $(25 \%)$ & Lanolin & 14 \\
Cinnamon $(80 \%)$ & Jojoba oil & 13 \\
& Lanolin & 22 \\
Cinnamon (50\%) & Jojoba oil & 24 \\
& Lanolin & 12 \\
Cinnamon (25\%) & Jojoba oil & 14 \\
Methylglyoxal (80\%) & Lanolin & 8 \\
& Jojoba oil & 4 \\
Methylglyoxal (50\%) & Lanolin & 21 \\
& Jojoba oil & 20 \\
& Lanolin & 10 \\
\hline
\end{tabular}

Cassia, at an $80 \%$ dilution in both jojoba oil and lanolin carrier oils, inhibited the growth of $P$. aeruginosa better than antibiotics amikacin and tobramycin (fig. 1). Cinnamon at the MIC in jojoba oil performed better than both amikacin and tobramycin and performed better than amikacin when diluted with lanolin. At the MIC in jojoba oil methylglyoxal in both carrier, oils performed nearly as well as amikacin and tobramycin in inhibiting the growth of $P$. aeruginosa. Other studies testing the efficacy of these essential oils, methylglyoxal, and other plant extracts have also demonstrated the success of their antimicrobial properties $[8,12,16]$. This study appears to be the first also to test the efficacy of the major component cinnamaldehyde for its role in the antimicrobial properties of essential oils cassia and cinnamon. The results of this isolated component of cassia and cinnamon oils at MIC were found to be more effective than the standard antibiotics (fig. 1).

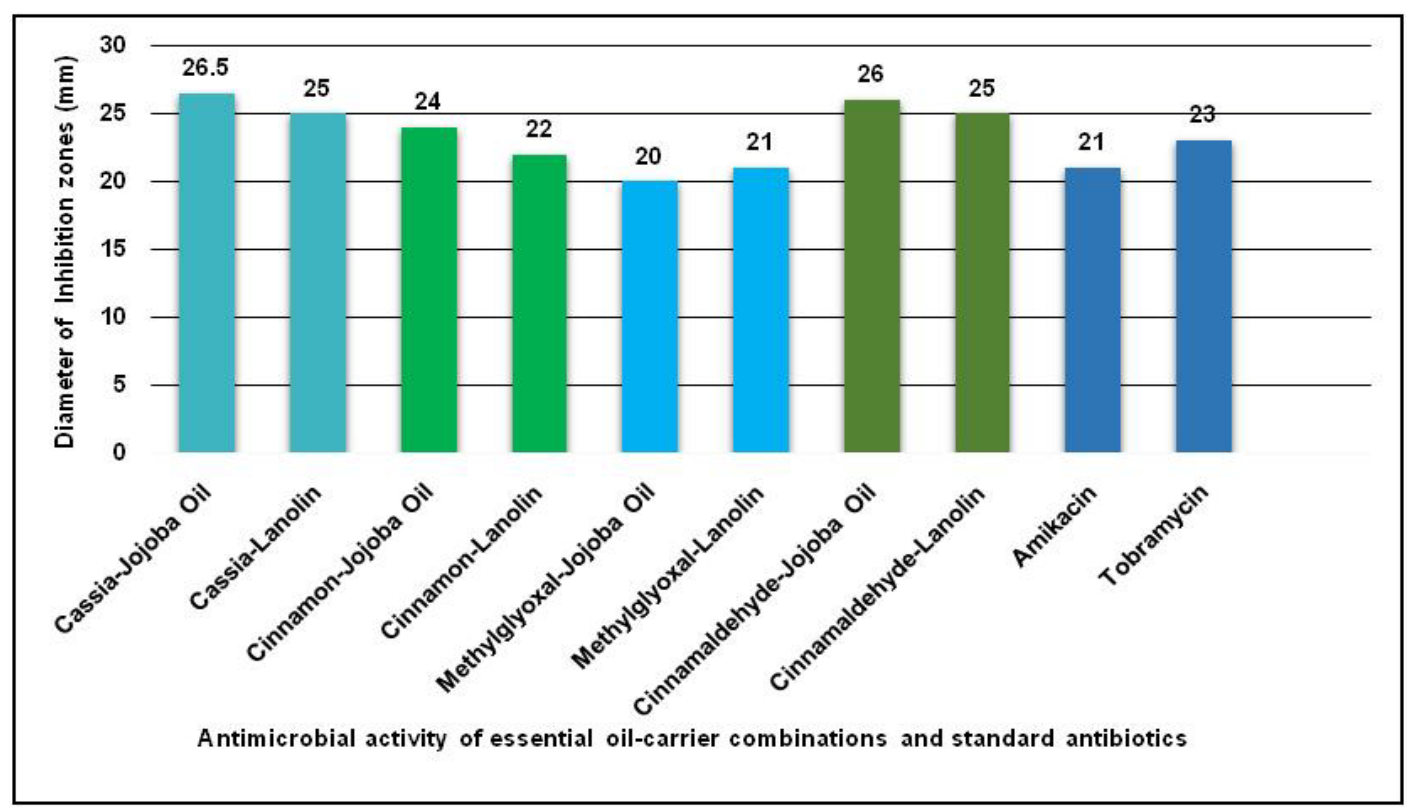

Fig. 1: Mean diameter of zone of inhibition $(\mathrm{mm})$ of $80 \%$ essential oil with $20 \%$ carrier oil with and standard antibiotics

\section{CONCLUSION}

In summary, this in vitro study demonstrated the potential of using emollients containing the essential oils, cassia and cinnamon bark, its major component cinnamaldehyde, as well as the major component of manuka honey, methylglyoxal for inhibiting $P$. aeruginosa infections. The results showed that the emollients tested could be a possible alternative treatment for $P$. aeruginosa applied topically (possibly).
Further research must be done to develop their clinical application and effectiveness with $P$. aeruginosa and other bacterial pathogens. Treatment of $P$. aeruginosa infections with essential oils could make a major global impact on treating hospital patients infected with this bacterium. This approach could also be used for bacterial infections $(P$. aeruginosa and other similar bacteria) in underdeveloped countries where access to antibiotics is limited. 


\section{ACKNOWLEDGEMENT}

We would like to thank Dr. Datta Naik, Professor of Chemistry, Monmouth University for his continuous and helpful commentary; Dr. Albert Rojtman of Jersey Shore University Medical Center for his collaboration and support. We also thank Mr. Kevin Young of dōTERRA for his support. Lastly, we thank the Summer Research Program and the Monmouth University Grant-in-Aid for Creativity for providing the funds to make this research project possible.

\section{CONFLICTS OF INTERESTS}

All authors have none to declare.

\section{REFERENCES}

1. Nazzaro F, Fratianni F, De Martino L, Coppola RC, De Feo V. Effect of essential oils on pathogenic bacteria. Pharmaceuticals 2013;6:1451-74.

2. Boire NA, Riedel S, Parrish NM. Essential oils and future antibiotics: new weapons against emerging 'superbugs? J Ancient Diseases Preventive Remedies 2013;2:1-4.

3. CDC. Antibiotic resistance threats in the united states. U. S. Dep Health Hum Serv Cent Dis Ctrl and Prev; 2013. p. 1-27.

4. Zaffiri L, Gardner J, Toledo-Pereyra LH. History of antibiotics. From salvarsan to cephalosporins. J Inv Sur 2012;25:67.

5. Davies J, Davies D. Origins and evolution of antibiotic resistance. Am Soc Microbiol 2010;74:417-33.

6. Anil C, Shahid RM. Antimicrobial susceptibility patterns of pseudomonas aerugionsa clinical isolates at a tertiary care hospital in Katahmandu, Nepal. Asian J Pharam Clin Res 2013;6:235-7.

7. The White House. National Strategy for Combating Antibiotic Resistant Bacteria. Washington, DC. The Center for Disease Control; 2015. p. 1-2.

8. Qureshi WK, Palayekar V, Dayan E, Mack J, Rojtman A. Combating the antibiotic resistance threat. Int J Pharm Pharm Sci 2015;7:68-72.
9. National Center for Infectious Diseases [Internet]. Atlanta: Centers for Disease Control and Prevention (US). Pseudomonas aeruginosa in healthcare settings; [about 2 screens]. Available from: https://www.cdc.gov/hai/organisms/pseudomonas.html. [Last accessed on $17 \mathrm{Feb} 2015]$.

10. Moore D [Internet]. Antibiotic classification and mechanism. Available from: http://dev.orthobullets.com/basic-science/9059/ antibiotic-classification-and-mechanism. [Last accessed on $27 \mathrm{Jul}$ 2015].

11. Planquette B, Timsit JF, Misset BY, Schwebel C, Azoulay E. Pseudomonas aeruginosa ventilator-associated pneumonia: predictive factors of treatment failure. Am J Respir Crit Care Med 2013;118:69-76.

12. Cooper RA, Halas E, Molan PC. The efficacy of honey in inhibiting strains of pseudomonas aeruginosa infected burns. J Burn Care Rehabil 2002;23:366-70.

13. Kavanaugh NL, Ribbeck K. Selecter antimicrobial essential oils eradicate pseudomonas spp. and staphylococcus aeureus biofilms. Appl Environ Microbiol 2012;78:4057-61.

14. Lawless, J. The illustrated encyclopedia of essential oils: The complete guide to the use of oils in aromatherapy and herbalism. New York: Barnes and Noble; 1995.

15. Bauer AW, Kirby WM, Sherris JC, Turck M. Antibiotic susceptibility testing by a standardized single disk method. Am J Clin Pathol 1966;45:493-6.

16. Santhanamari $\mathrm{T}$, Meenakshi $\mathrm{PR}$, Velayutham $\mathrm{S}$. In vitro antibacterial activity of extracts of lawsonia inermis and punica granatum against clinically isolaticed antiobiotic resistant pseudomonas aeuruginosa and staphylococcus aureus. Asian J Pharm Clin Res 2011;4:62-4.

\section{How to cite this article}

- Jenies Grullon, James P Mack, Albert Rojtman. Using essential oils to combat the threat of multi-drug resistant bacteria, Pseudomonas aeruginosa. Int J Pharm Pharm Sci 2016;8(12):180-183. 